\title{
2020 Virtual MRS Spring/Fall Meeting a successful, valuable experience
}

$\mathrm{W}$ ith more than 6000 participants from 61 countries and 280 virtual events, the 2020 Virtual Materials Research Society (MRS) Spring/Fall Meeting, held from November 27 to December 4, proved to be not only a new venture but a successful, valuable experience as well. Experts from around the world presented talks, members networked online, and MRS staff learned how to host virtual poster sessions and live panel discussions.

The move to a virtual format came from an abundance of caution because of the ongoing global COVID-19 pandemic. The health and well-being of our members, staff, and the materials community are of the utmost importance to MRS and guided this decision.

New methodologies were used to present the quality of content MRS members are accustomed, including tutorials, exhibits, the Women in Materials Science \& Engineering program, student-led events, awards recognition, Science as Art, and MRS TV. This also allowed the opportunity to find creative ways to make traditional in-person events and experiences meaningful and engaging in a different format. Another benefit was that even after the meeting concluded, members could continue to access and listen to all recorded talks through the end of December 2020, expanding the ability of meeting attendees to hear additional talks that busy schedules typically do not permit while attending in person.

The virtual meeting also allowed the option of live or pre-recorded presentations, further accommodating the differences in time zones and schedules. Electronic scheduling included reminders of upcoming events of interest to increase attendance; Q\&A features permitted attendees to engage during presentations; and the option to send electronic meeting invitations increased opportunities for networking. An overview from a numeric perspective is almost 713,000 desktop page views, $\sim 300,000$ banner ad views, close to 10,000 public messages, 5300 private messages, and close to 80,000 mobile page views.

Congratulations go to the 2020 Spring Meeting Chairs Qing Cao, Miyoung Kim, Rajesh Naik, James M. Rondinelli, and Hong Wang, and the 2020 Fall Meeting Chairs Michael E. Flatté, Michael P. Rowe, Sabrina Sartori, Prasad Shastri, and Chongmin Wang for putting together an excellent technical program along with various special events. They organized
14 topical clusters, adhering to the same breadth of sessions and quality of presentations expected of MRS meetings.

Targeted professional development video content remains available OnDemand to members, including "How to Land a Faculty Position," "Writing Concrete Abstracts and Titles," "Career Planning in a Crisis," "Virtual Interviewing and the Job Talk," "Essentials of Getting Your Work Published," and more.

On the lighter side were virtual magic shows and a selfie booth, both well received and attended. MRS Meeting Scene ${ }^{\circledR}$ provided daily highlights and news during the conference.

Plans are well underway for the 2021 Virtual MRS Spring Meeting \& Exhibit, April 17-23. Members can expect an impressive program of

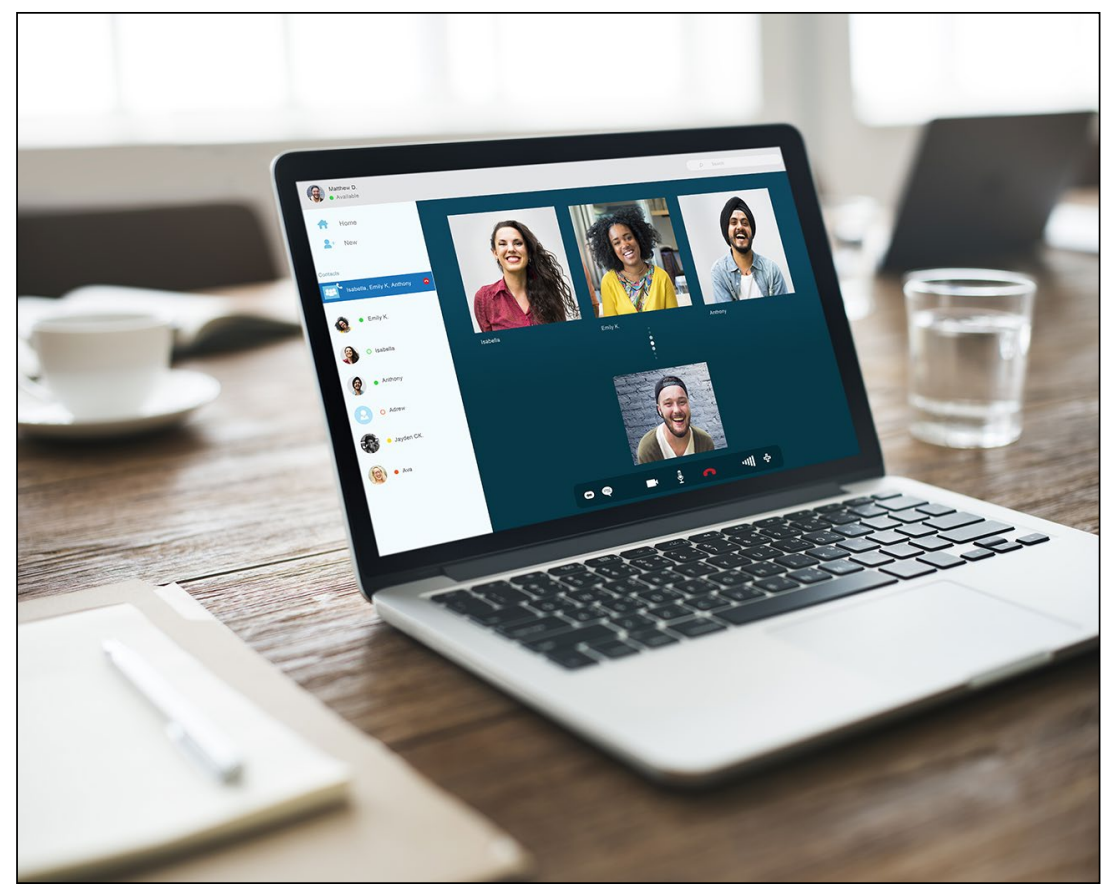


the latest materials research, including technical symposia covering emerging electronic materials, quantum materials, biomaterials, artificial intelligence, and sustainability. Networking opportunities with fellow attendees and presenters worldwide will help in the sharing of ideas. A virtual exhibit hall will feature the latest products, solutions, and applications to help further research. In addition, professional development events will help to elevate career-building skills. Registration is now open.

Multidisciplinary research has proven essential to innovation. The Spring Meeting will allow an opportunity for researchers working in

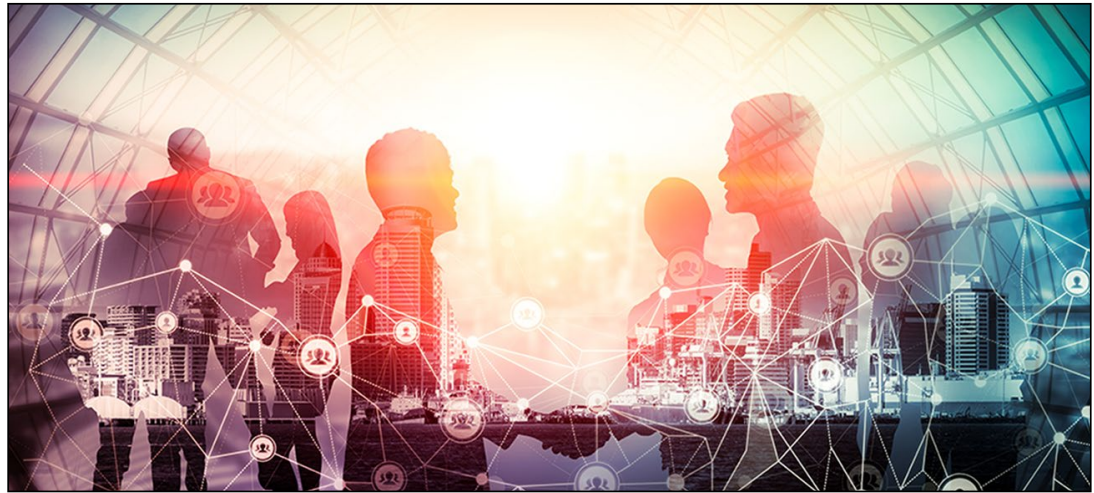

seemingly unrelated fields to gather virtually to promote, share, and discuss issues and developments across disciplines. It provides researchers-from students and postdoctoral fellows, to Nobel and Kavli Prize laureates - with a chance to exchange technical information and network with colleagues. We look forward to "seeing" you soon.

\section{Thank you!' to our 2020 MRS Spring Meeting Symposium Supporters!}

\section{PLATINUM}

National Science Foundation GOLD

Los Alamos National Laboratory National Science Foundation Office of Naval Research Science Advances I AAAS SILVER

Corning Research and Development Corporation: Thin Films and Surfaces
Daicel Corporation

Neware Technology Limited

Taiyo Nippon Sanso

Wuhan LAND Electronics Co., Ltd BRONZE

ACS Nano I ACS Publications

Alnylam Pharmaceuticals

Applied Diamond Inc.

Applied Physics Letters I AIP Publishing

BAE Systems
Colorado School of Mines Chemistry Department

DCA Instruments

Future Semiconductor Business

Gatan, Inc.

King Abdullah University of Science and Technology

Kyma Technologies, Inc.

MilliporeSigma

Neware Technology LLC
NovaCentrix

Royal Society of Chemistry

Rubius Therapeutics

Sandia National Laboratory

Sandia National Laboratory-08811

Energy Storage

Scienta Omicron, Inc.

Southwest Family Dental

Ultrafast Systems

Vigor Tech USA

\section{Thank you! to our 2020 MRS Fall Meeting Symposium Supporters!}

\section{PLATINUM}

National Science Foundation - Award \#2028092

Office of Naval Research

Perkin Elmer

Samsung SDI

GOLD

National Science Foundation

Science | AAAS

SILVER

Carl Zeiss Microscopy, LLC
ETH Zürich

Lam Research

Materials Today | Elsevier

PicoQuant

Science Robotics | AAAS

University of Duisburg-Essen, CENIDE

BRONZE

ACS Energy Letters | ACS

Publications
ACS Photonics | ACS Publications

Allevi, Inc

CAMECA Instruments, Inc.

General Electric Research

Goodfellow Corporation

Kleindiek Inc.

MilliporeSigma

Oxford PV

Pittsburgh Quantum Institute (University of Pittsburgh)
Polytechnique Montréal Chemical Engineering Department

Sandia National Laboratories The Journal of Physical Chemistry Letters | ACS Publications Thermo Fisher Scientific Two-Dimensional Crystal Consortium Materials Innovation Platform (2DCC-MIP) University of Auckland 